\title{
Safety and efficacy of intravitreal conbercept injection after vitrectomy for the treatment of proliferative diabetic retinopathy
}

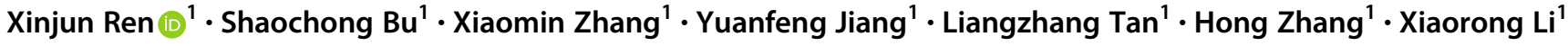

Received: 8 May 2018 / Revised: 2 January 2019 / Accepted: 18 February 2019 / Published online: 14 March 2019

(c) The Royal College of Ophthalmologists 2019

\begin{abstract}
Background/objectives The aim of this study was to evaluate the safety and efficacy of intravitreal conbercept (a recombinant fusion protein that primarily targets vascular endothelial growth factors) after vitrectomy for the management of proliferative diabetic retinopathy without tractional retinal detachment (TRD).

Subjects/methods Fifty patients with non-clearing vitreous haemorrhage (VH) due to proliferative diabetic retinopathy without TRD were enroled. They were randomly divided into control and treatment groups ( 25 eyes to each group) after they provided informed consent. The treatment group received intravitreal conbercept $(10 \mathrm{mg} / \mathrm{mL}, 0.5 \mathrm{mg})$ immediately after surgery, while the control group did not. The best corrected visual acuity (BCVA) and the central retinal thickness were measured.

Results There were no significant between-group differences in baseline characteristics $(P>0.05)$, except in age $(P=$ 0.003). Improvement in BCVA was significantly greater at $1,4,12$, and 24 weeks post surgery in the treatment group than it was in the control group $(P<0.001)$. There were more cases in the control group who developed recurrent $\mathrm{VH}$, but the recurrence rate of $\mathrm{VH}$ was not significantly different between the two groups at 12 and 24 weeks post surgery $(P=0.192$ and 0.103). Central retinal thickness was lower in the treatment group than in the control group at 1 week $(P=0.012)$, 4 weeks $(P=0.01), 12$ weeks $(P=0.001)$, and 24 weeks $(P=0.004)$ post surgery, which were statistically significant. Conclusions An intravitreal injection of conbercept after vitrectomy improved visual acuity and seemed to reduce the recurrence of $\mathrm{VH}$ resulting in prompt visual recovery in the PDR patients.
\end{abstract}

\section{Introduction}

Diabetic retinopathy (DR) is one of the most common causes of irreversible blindness in China due to the rising economy and changes in modern lifestyle. In 2010, Yang et al. estimated the prevalence of diabetes in the Chinese population to be $9.7 \%$, accounting for 92.4 million adults posing a significant public health issue $[1,2]$. The main causes of vision loss are diabetic macular oedema, diabetic optic neuropathy, retinal undefined, vitreous undefined (vitreous haemorrhage; $\mathrm{VH}$ ), traction retinal detachment

These authors contributed equally: Hong Zhang, Xiaorong Li

Xiaorong $\mathrm{Li}$

xiaorli@163.com

1 Tianjin Medical University Eye Hospital \& Tianjin Medical University Eye Institute, Tianjin, China
(TRD), and neovascular glaucoma [3, 4]. For early-stage proliferative DR (PDR), where no TRD is detected by ultrasonography, timely pan-retinal photocoagulation (PRP) can reduce the rate of retinal neovascularization as well as the extent of the perfusion-free areas, thereby stabilizing the condition [5]. Furthermore, in DR with non-clearing vitreous undefined 1-4 months from onset, the DRVS study has demonstrated significantly better visual outcome with vitrectomy combined with intraocular retinal photocoagulation [6]. However, the final outcome for patients with type II diabetes were hindered by the complications of diabetic macular oedema and the recurrence of $\mathrm{VH}$, etc. Although surgical techniques are constantly evolving, the dissection of the fibrovascular membranes remains technically challenging. It is difficult to remove the fibrovascular membranes completely during surgery. Especially the neovascularization presents on the optic disc surface and the vicinity of the incision sites. Additionally, a large proportion of patients in our practice do not receive PRP before the bleeding occurs. This is quite a different process from many 
other countries that have screening programmes/regular patient reviews. Furthermore, intraoperative laser photocoagulation can aggravate macular oedema and inflammation after the surgery, which further deteriorate the postoperative visual outcome [7].

Vascular endothelial growth factor (VEGF) plays an important role in the treatment of DR. The purpose of the intravitreal application of VEGF inhibitors are two folds: (1) to induce the regression of retinal neovascularization; and (2) to reduce the vascular permeability resulting in the resolution of diabetic macular oedema [8-11]. VEGF inhibitors may reduce the occurrence of intraoperative and postoperative complications and rapidly improve recurrent neovascular leakage [12-14]. These agents can also alleviate PDR-associated $\mathrm{VH}$ in the short term and rapidly reduce neovascularization of the iris and retina [15]. Bevacizumab is the most commonly used anti-VEGF agent, and is employed as an adjunctive treatment to vitrectomy for PDR. It has demonstrated benefits for postoperative visual function and recurrent $\mathrm{VH}$ [10]. Additionally, various VEGF inhibitors have been reported to improve the vision in eyes with diabetic macular oedema [16].

Conbercept (KH902; Chengdu Kanghong Biotech Co., Ltd., Sichuan, China) was made available for clinical application as an inhibitor of the VEGF-A, VEGF-B, and placental growth factor receptors [17, 18]. Our group observed a significant regression of retinal neovascularization in patients with intravitreal conbercept injection prior to the vitrectomy [19]. Li et al. reported that the conbercept was effective in the treatment of DME. The improvement of the vision was demonstrated at 1 month after the injection [20]. Therefore, we performed this interventional, controlled trial to investigate the safety and effect of adjunctive intravitreal conbercept immediately after $25-\mathrm{G}$ vitrectomy for the management of VH due to PDR without TRD.

\section{Subjects and methods}

The study was conducted in accordance with the Declaration of Helsinki, permission was granted by the Ethics Committee at Tianjin Medical University, and all patients provided written informed consent. This study was registered with clinicaltrials.gov (ID NCT03426540). We enroled 50 eyes from 50 patients that were affected by $\mathrm{VH}$ due to PDR from January 2017 through March 2018. Patients with non-clearing VH and fibrovascular proliferation without TRD who underwent pars plana vitrectomy (PPV) at Tianjin Medical University Eye Hospital were included in the study. The exclusion criteria were: a history of previous PPV; stroke, a thromboembolic event within 6 months prior to the surgery, myocardial infarction in the previous 6 months contraindicating the withdrawal of anti- platelet and anti-coagulant medications. Patients who required silicone oil due to intraoperative complications (e.g., severe bleeding or iatrogenic retinal break) or a particularly long or complex surgery were excluded from this study.

All patients received a thorough explanation of the study design and aim, informed consent, and consent to publish were obtained. Patients who were being treated with a $25-\mathrm{G}$ vitrectomy were randomly selected according to randomized table to administer intravitreal injection of conbercept or not (25 patients each group). Baseline patient characteristics are summarized in Table 1.

Three-port 25-G PPV was performed in all patients using a Constellation system (Alcon, Fort Worth, TX, USA) at a speed of 5000 cuts per minute. Fibrovascular tissues and opacified vitreous fluid as well as blood clots adherent to the vitreous base were removed. Intraoperative endolaser PRP was used at the end of the surgery. Finally, an intraocular tamponade with gas was performed if necessary. We decided to perform the tamponade based on the difficulty and complexity of the surgery. Patients who required silicone oil due to intraoperative complications (e.g., severe bleeding or iatrogenic retinal break) or a particularly long or complex surgery were excluded from this study. All surgical procedures described in this study were performed by one surgeon (HZ, Tianjin Medical University Eye Hospital), who was masked from information during the vitrectomy regarding whether the patients would undergo intravitreal conbercept injection at the end of the surgery.

Patients in the treatment group received an intravitreal injection of conbercept $(10 \mathrm{mg} / \mathrm{mL}, 0.5 \mathrm{mg})$ in the inferotemporal quadrant $3.5-4 \mathrm{~mm}$ from the sclerocorneal

Table 1 Baseline clinical preoperative characteristics

\begin{tabular}{llll}
\hline Characteristics & $\begin{array}{l}\text { Group treated } \\
\text { with conbercept }\end{array}$ & $\begin{array}{l}\text { Group without } \\
\text { conbercept }\end{array}$ & $P$ value \\
\hline Number of cases & 25 & 25 & NA \\
Number of eyes & 25 & 25 & NA \\
Gender (male/ & $16 / 9$ & $14 / 11$ & 0.564 \\
female) & & $62(46-80)$ & 0.003 \\
Age (years) & $57(28-69)$ & $12.04 \pm 6.05$ & 0.258 \\
Duration of DM $(n)$ & $10.36 \pm 4.17$ & & \\
Lens status (n) & & 17 & 0.529 \\
Phakic eye & 19 & 8 & 0.529 \\
Pseudophakic eye & 6 & & \\
Type of DM (n) & & 0 & NA \\
Type 1 (IDDM) & 0 & 25 & NA \\
Type 2 (NIDDM) & 25 & $17 / 8$ & 0.248 \\
Prior PRP (yes/no) & $13 / 12$ & &
\end{tabular}

$D M$ diabetes mellitus, IDDM insulin-dependent diabetes mellitus, NIDDM noninsulin-dependent diabetes mellitus, $P R P$ pan-retinal photocoagulation, $N A$ not available 
limbus using a sterile technique after the 25-G vitrectomy, while those in the control group did not. All intravitreal injections were performed by the same surgeon (HZ). Because PDR is a multifactorial pathology, and the functional results of the surgery are depended on a series of preoperative and intraoperative factors, we assigned to the following preoperative parameters a score from 0 to 3 , in order to obtain two homogeneous groups of surgical complexity according to the previous published literatures, which were listed in Table 2 [21]. All patients received eye drops containing antibiotics and dexamethasone with tapered frequency during the 4 weeks' period after surgery.

\section{Examination}

The primary outcome measures included any changes in best corrected visual acuity (BCVA), central retinal thickness, and VH recurrence. Pre- and postoperative examinations included a detailed medical history, BCVA test using a Landolt $\mathrm{C}$ acuity chart, and slit-lamp biomicroscopy with careful evaluation for the presence of $\mathrm{VH}$. Complete ophthalmic examinations and colour fundus photography were performed at baseline and at 1,4 , and 12 weeks post surgery. Ultrasonography was performed at baseline and at 4 weeks after the injection. Preoperative fluorescein angiogram was not performed in the majority of cases due to the presence of moderate-to-severe $\mathrm{VH}$, which prevented an adequate view of the fundus. BCVA was analysed on a logarithm of minimal angle of resolution (logMAR) scale; "counting fingers" vision was defined as 0.01 (2.0 logMAR) and "hand movement" was defined as 0.001 (3.0 logMAR) [22]. Visual improvement was defined as an increase of at least $0.3 \operatorname{logMAR}$ units. Retinal thickness and VH recurrence were analysed by optical coherence tomography and B-ultrasound. Intraoperative evaluations included the formation of iatrogenic retinal tears, the need for silicone oil endotamponade, and the duration of surgery. These parameters were used to evaluate whether conbercept could be used after vitrectomy. At each follow-up visit, colour fundus photography made it possible to quantify the amount of $\mathrm{VH}$.

Statistical calculations were performed using the SPSS software package. Data are presented as the mean \pm standard deviation. $T$-tests, Pearson chi-square tests, chi-square tests with Yates' correction, or Fisher's exact tests were performed to assess between-group differences. A $P$-value $<0.05$ was considered statistically significant.

\section{Results}

The BCVA results were showed in Table 3. Improvements in corrected visual acuity were stastistically significant in the treatment group compare that in the control group at different time points $(P<0.001)$.

PPV was successfully performed in both groups. Minor bleeding during surgery is common and thus was not reported for our analysis. Twenty-three patients $(92 \%)$ in the experimental group and 22 patients $(88 \%)$ in the control group received intraoperative PRP $(P=1.0)$. Fluid air exchange and endotamponade with sterile air were performed in 5 patients $(20.0 \%)$ in the treatment group and 4 patients $(16.0 \%)$ in the control group $(P=0.731)$. The mean surgical time was $42.04 \pm 7.48 \mathrm{~min}$ in the treatment group and $43.92 \pm 7.50 \mathrm{~min}$ in the control group $(P=0.379)$.
Table 2 Baseline clinical preoperative characteristics

\begin{tabular}{|c|c|c|c|c|}
\hline & \multicolumn{2}{|c|}{ Group without conbercept } & \multicolumn{2}{|c|}{ Group treated with conbercept } \\
\hline & $\begin{array}{l}\text { Cases }(25 \\
\text { patients) }\end{array}$ & $\begin{array}{l}\text { Complexity surgery } \\
\text { score }\end{array}$ & $\begin{array}{l}\text { Cases }(25 \\
\text { patients) }\end{array}$ & $\begin{array}{l}\text { Complexity surgery } \\
\text { score }\end{array}$ \\
\hline \multicolumn{5}{|l|}{ Vitreous haemorrhage } \\
\hline Absent $(0)$ & 0 & - & 0 & - \\
\hline Mild (+1) & 4 & 4 & 3 & 3 \\
\hline Moderate $(+2)$ & 12 & 24 & 10 & 20 \\
\hline Severe $(+3)$ & 9 & 27 & 12 & 36 \\
\hline \multicolumn{5}{|c|}{ Amount of previous retinal photocoagulation } \\
\hline Complete PRP (0) & 2 & - & 3 & - \\
\hline $\begin{array}{l}\text { Incomplete PRP } \\
(+1)\end{array}$ & 5 & 5 & 4 & 4 \\
\hline Focal $(+2)$ & 6 & 12 & 7 & 14 \\
\hline None $(+3)$ & 12 & 36 & 11 & 33 \\
\hline $\begin{array}{l}\text { Total complexity } \\
\text { surgery score }\end{array}$ & 108 & & 110 & \\
\hline
\end{tabular}


Table 3 Visual acuity levels at preoperative and final visit in patients with early PDR with and without conbercept after vitrectomy
Table 4 Recurrence of vitreous haemorrhage in two groups at different time after operation

\begin{tabular}{llll}
\hline BCVA & Group treated with conbercep & Group without conbercept & $P$ value \\
\hline Preoperative BCVA & $1.51 \pm 0.70$ & $1.54 \pm 0.83$ & 0.883 \\
Postoperative 1 week & $0.32 \pm 0.15$ & $0.64 \pm 0.31$ & $<0.001$ \\
Postoperative 1 month & $0.31 \pm 0.15$ & $0.61 \pm 0.29$ & $<0.001$ \\
Postoperative 3 months & $0.29 \pm 0.14$ & $0.59 \pm 0.30$ & $<0.001$ \\
Postoperative half year & $0.29 \pm 0.16(n=23)$ & $0.64 \pm 0.35(n=24)$ & $<0.001$ \\
\hline
\end{tabular}

$P D R$ proliferative diabetic retinopathy, $B C V A$ best corrected visual acuity

\begin{tabular}{llll}
\hline VH recurrence & $\begin{array}{l}\text { Group treated with conbercept }\left(n=\begin{array}{l}\text { Group without conbercept }(n= \\
25)\end{array}\right. \\
\text { 25) }\end{array}$ & $P$ value \\
\hline Postoperative 1 week & 0 & 0 & NA \\
Postoperative 1 month & 0 & $1(4.0 \%)$ & 1.0 \\
Postoperative 3 months & $1(4.0 \%)$ & $5(20.0 \%)$ & 0.192 \\
Postoperative 6 months & $1(4.0 \%)$ & $6(24.0 \%)$ & 0.103 \\
\hline
\end{tabular}

$V H$ vitreous haemorrhage, $N A$ not available
Although the rate of recurrent $\mathrm{VH}$ was lower in the treatment group than in the control group at 12 weeks and 24 weeks post surgery, this difference was not statistically significant $(P=0.192$ and 0.103$)$. The complete results are summarized in Table 4. In the control group, recurrent $\mathrm{VH}$ occurred in $4.0 \%$ of eyes ( 1 of 25$)$ at 4 weeks post surgery, $20.0 \%$ of eyes ( 5 of 25 ) at 12 weeks post surgery, and $24 \%$ of eyes (6 of 25, cumulative calculation) at 24 weeks post surgery. In the treatment group, recurrent $\mathrm{VH}$ occurred in $0 \%$ of eyes at 4 weeks post surgery, $4.0 \%$ of eyes (one of 25 ) at 12 weeks post surgery, and $4 \%$ of eyes ( 1 of 25 , cumulative calculation) at 24 weeks post surgery. Conbercept injections were well tolerated in all patients; there were no reported postoperative side effects such as uveitis, endophthalmitis, ocular toxicity, myocardial infarction, or obvious systemic adverse effects.

In the control group, central retina thickness was $291 \pm$ $46 \mu \mathrm{m}$ at 1 week post surgery, $279 \pm 40 \mu \mathrm{m}$ at 4 weeks post surgery, $281 \pm 36 \mu \mathrm{m}$ at 12 weeks post surgery, and $267 \pm$ $31 \mu \mathrm{m}$ at 24 weeks post surgery. In the treatment group, central retinal thickness was $258 \pm 45 \mu \mathrm{m}$ at 1 week post surgery, $249 \pm 41 \mu \mathrm{m}$ at 4 weeks post surgery, $242 \pm 36 \mu \mathrm{m}$ at 12 weeks post surgery, and $238 \pm 33 \mu \mathrm{m}$ at 24 weeks post surgery. Central retinal thickness was significantly lower in the treatment group than in the control group at each time point $(P=0.012,0.01,0.001$, and 0.004 , respectively, Table 5).

All patients were examined within the first week. Ocular hypotension occurred after surgery in five cases $(20.0 \%)$ in the control group and in six cases $(24.0 \%)$ in the treatment group $(P=0.733)$. Intraocular pressure (IOP) fluctuated between 6 and $8 \mathrm{~mm} \mathrm{Hg} \mathrm{1-5} \mathrm{days} \mathrm{post} \mathrm{surgery.} \mathrm{Choroid}$ detachment was not reported in either group. Anterior chamber inflammation occurred during the early postoperative period in four eyes $(16 \%)$ in the control group and in three eyes $(12 \%)$ in the treatment group $(P=1.0)$ and resolved within 1 week after topical application of mydriatic, steroidal, and non-steroidal eye drops. Ocular hypertension occurred after surgery in five cases $(20.0 \%)$ in the control group and in two cases $(8.0 \%)$ in the treatment group $(P=0.415)$. IOP fluctuated between 23 and $35 \mathrm{~mm}$ Hg 1-2 weeks post surgery. Systemic and topical administration of IOP-lowering drug medications controlled the IOP effedtively. There were no reports of proliferative vitreoretinopathy, retinal detachment, rubeosis iridis, or neovascular glaucoma post surgery.

\section{Discussion}

In the current study, our results showed that the intravitreal injection of conbercept at the end of the vitrectomy for PDR without tractional retinal detachment resulted in a better postoperative visual acuity, thinner central retinal thickness and a trend to reduced $\mathrm{VH}$ recurrences. Thus, a prompt visual functional recovery could be achieved.

The intravitreal injection of conbercept at the end of the surgery could prevent the progression of neovascularization period without the concern of the progression of fibrocellular membrane contraction in the early postoperative period. Vitrectomy combined with PRP has been reported to be one of the most effective approaches to preserve vision in the treatment of PDR. However, complications, such as intraoperative bleeding, are inevitable [1, 23, 24]. 
Table 5 Comparison of central retinal thickness at different time after operation in two groups

\begin{tabular}{llll}
\hline Central retinal thickness $(\mu \mathrm{m})$ & Group treated with conbercept & Group without conbercept & $P$ value \\
\hline Postoperative 1 week & $258 \pm 45$ & $291 \pm 46$ & 0.012 \\
Postoperative 1 month & $249 \pm 41$ & $279 \pm 40$ & 0.01 \\
Postoperative 3 months & $242 \pm 36$ & $281 \pm 36$ & 0.001 \\
Postoperative half year & $238 \pm 33(n=24)$ & $267 \pm 31(n=23)$ & 0.004 \\
\hline
\end{tabular}

Therefore, methods for reducing the retinal neovascular membrane and controlling the proliferative process in patients with PDR are critical to successful treatment. Previous studies demonstrated that the use of anti-VEGF drugs in PDR reverses neovascularization of the retina and iris [25], induces vascular endothelial cell apoptosis and new vessel occlusion or disappearance, increases pericytes numbers, promotes angiogenesis and vascular maturation to decrease fundus exudation and bleeding, and maintains stability of the blood retinal barrier [26].

In our previous study, we investigated the utility of preoperative intravitreal injections of conbercept for improving the safety and effectiveness of vitrectomy in eyes with severe PDR. Inhibiting the growth and spread of neovascular processes and reducing vascular permeability decreased the rate of intraoperative complications [19]. In certain cases, it is difficult to remove the neovascular membrane completely during surgery, especially the parts located on the optic disc surface or peripheral retina. These residual pathologies can potentially lead to $\mathrm{VH}$ recurrence, neovascular glaucoma, or TRD, necessitating multiple surgeries. Additionally, a large proportion of patients in our cohort did not receive PRP prior to surgery, as intraoperative laser photocoagulation ( $92 \%$ in the experimental group and $88 \%$ in the control group) can aggravate macular oedema and inflammation. The intravitreal injection of conbercept could reduce vascular permeability, decrease retinal thickness and congestion, and to be beneficial for the recovery of retinal function in the post operation. Intravitreal conbercept injection may be beneficial for retinal reattachment and PRP as well as the early control of disease progression in patients with PDR [19]. The intravitreal conbercept at the end of the surgery qualitatively reduced the recurrence of $\mathrm{VH}$ and definitively improved the effectiveness of PPV treatment and postoperative visual acuity in our study.

It can be inferred that a single intravitreal injection of an anti-VEGF agent produces transient rather than sustained effects on retinal and optic disc neovascularization [27]. We observed a trend in the reduction of the $\mathrm{VH}$ recurrence in our study. But the difference was not statistically significant. Some studies have reported increased fibrous proliferation and TRD after intravitreal injection of antiVEGF preparations [28]. Neovascularization may be replaced by fibrous tissue, such that TRD and VH occur secondary to fibrous tissue contraction [29]. Arevalo et al. [30] reported further deterioration and progression of TRD 13 days after intravitreal injection of anti-VEGF treatment. Ishikawa et al. [31] suggested that the number of fibres and extent of proliferation, as well as adhesion of the fibrous vascular membrane, were increased 7 days after intravitreal injection of anti-VEGF treatment. Significant regression of neovascularization was achieved 10 days after the injection [32, 33]. In this study, we performed intravitreal conbercept injections after vitrectomy, which could reduce the possibility of membrane contraction that is induced by conbercept due to the removal of the fibrocellular membranes. Therefore, the retinal traction was alleviated. Thus, we speculate that injection after vitrectomy could not only avoid TRD and VH secondary to the contraction of fibrous tissues but also decrease angiogenesis and stabilize the vascular permeability. Indeed, no fibrous proliferation or TRD occurred post surgery in our study.

For severe PDR, preoperative intravitreal conbercept injection is intended to facilitate surgery. However, for early-stage PDR, intravitreal injection of conbercept after vitrectomy can play a more important role in accelerating the process of vision recovery and reducing the macular oedema. These conclusions can also be generalized to other anti-VEGF agents because of the same underlying mechanism, such as the anti-VEGF fusion protein and monoclonal antibody anti-VEGF drugs since these agents have been shown to be effective in the suppression of new vessels and reduction of macular oedema.

The present study had several important limitations. Although we enroled subjects prospectively, the sample size was relatively small, and the duration of follow-up was short. These flaws may have led to bias or error in the experimental results. Despite these limitations, our results indicate that postoperative intravitreal injection of conbercept is safe and effective for the adjunct treatment of early-stage PDR combined with VH. Intravitreal conbercept seemed to reduce the recurrence of $\mathrm{VH}$, although the difference was not significant in our study; moreover, it preserved postoperative visual acuity. Future studies are required to confirm our results indicating postoperative intravitreal conbercept as a tool for improving retinal function and quality of life in patients with PDR. 


\section{Summary}

\section{What was known before}

- For the management of PDR, it is difficult to completely remove the fibrovascular membranes during surgery, especially from the optic disc surface or from the periphery, extreme periphery, or vicinity of the incision sites; thus, repeated operations may be required. Furthermore, intraoperative laser photocoagulation can aggravate macular oedema and inflammation after the surgery.

\section{What this study adds}

- In the current study, our results showed that the intravitreal injection of conbercept at the end of the vitrectomy for PDR without tractional retinal detachment resulted in a better postoperative visual acuity, thinner central retinal thickness and less frequent $\mathrm{VH}$ recurrences

- Thus, a prompt visual functional recovery could be achieved.

Acknowledgements The authors thank Fuhua Yang for help with the statistical analysis.

Funding This article is supported by National Natural Science Foundation of China (81600723).

\section{Compliance with ethical standards}

Conflict of interest The authors declare that they have no conflict of interest.

Publisher's note: Springer Nature remains neutral with regard to jurisdictional claims in published maps and institutional affiliations.

\section{References}

1. Crawford TN, Alfaro DV, Kerrison JB, Jablon EP. Diabetic retinopathy and angiogenesis. Curr Diabetes Rev. 2009;5:8-13.

2. Yang W, Lu J, Weng J, Jia W, Ji L, Xiao J, et al. Prevalence of diabetes among men and women in China. $\mathrm{N}$ Engl $\mathrm{J}$ Med. 2010;362:1090-101.

3. Ehrlich R, Harris A, Ciulla TA, Kheradiya N, Winston DM, Wirostko B. Diabetic macular oedema: physical, physiological and molecular factors contribute to this pathological process. Acta Ophthalmol. 2010;88:279-91.

4. Liu L, Wu X, Liu L, Geng J, Yuan Z, Shan Z, et al. Prevalence of diabetic retinopathy in mainland China: a meta-analysis. PLoS ONE. 2012;7:e45264.

5. Subash M, Comyn O, Samy A, Qatarneh D, Antonakis S, Mehat $\mathrm{M}$, et al. The effect of multispot laser panretinal photocoagulation on retinal sensitivity and driving eligibility in patients with diabetic retinopathy. JAMA Ophthalmol. 2016;134:666-72.

6. The Diabetic Retinopathy Vitrectomy Study Research Group. Early vitrectomy for severe vitreous hemorrhage in diabetic retinopathy. Two-year results of a randomized trial. Diabetic Retinopathy Vitrectomy Study report 2. Arch Ophthalmol. 1985;103 (11):1644-52.

7. Farouk MM, Naito T, Sayed KM, Nagasawa T, Katome T, Radwan $\mathrm{G}$, et al. Outcomes of 25-gauge vitrectomy for proliferative diabetic retinopathy. Graefes Arch Clin Exp Ophthalmol. 2011;249:369-76.

8. Aoki Y, Tomith M, Sato T, Watanabe M, Kase H, Fujita K, et al. Neoadjuvant chemotherapy for patients younger than 50 years with high-risk squamous cell carcinoma of the cervix. Gynecol Oncol. 2001;83:263-7.

9. Martinez-Zapata MJ, Marti-Carvajal AJ, Solà I, Pijoán JI, BuilCalvo JA, Cordero JA, et al. Anti-vascular endothelial growth factor for proliferative diabetic retinopathy. Cochrane Database Syst Rev. 2014;11:CD008721.

10. Pakzad-Vaezi K, Albiani DA, Kirker AW, Merkur AB, Kertes PJ, Eng KT, et al. A randomized study comparing the efficacy of bevacizumab and ranibizumab as pre-treatment for pars plana vitrectomy in proliferative diabetic retinopathy. Ophthalmic Surg Lasers Imaging Retin. 2014;45:521-4.

11. Storkebaum E, Lambrechts D, Canneliet P. VEGF: once regarded as a specific angiogenic factor, now implicated in neuroprotection. Bioessays. 2004;26:943-54.

12. El-Batarny AM. Intravitreal bevacizumab as an adjunctive therapy before diabetic vitrectomy. Clin Ophthalmol. 2008;2:709-16.

13. Farahvash MS, Majidi AR, Roohipoor R, Ghassemi F. Preoperative injection of intravitreal bevacizumab in dense diabetic vitreous hemorrhage. Retina. 2011;31:1254-60.

14. Jorge R, Costs RA, Calucci D, Cintra LP, Scott IU. Intravitreal bevacizumab (Avastin) for persistent new vessels in diabetic retinopathy (IBEPE study). Retina. 2006;26:1006-13.

15. Bakri SJ, Donaldson MJ, Link TP. Rapid regression of disc neovascularization in a patient with proliferative diabetic retinopathy following adjunctive intravitreal bevacizumab. Eye. 2006;20:1474-5.

16. Diabetic Retinopathy Clinical Research N, Wells JA, Glassman AR, Ayala AR, Jampol LM, Aiello LP, et al. Aflibercept, bevacizumab, or ranibizumab for diabetic macular edema. $\mathrm{N}$ Engl $\mathbf{J}$ Med. 2015;372:1193-203.

17. Shinkai A, Ito M, Anazawa H, Yamaguchi S, Shitara K, Shibuya M. Mapping of the sites involved in ligand association and dissociation at the extracellular domain of the kinase insert domaincontaining receptor for vascular endothelial growth factor. J Biol Chem. 1998;273:31283-8.

18. Zhang ZH, Liu HY, Hernandez-Da Mota SE, Romano MR, Falavarjani KG, Ahmadieh $\mathrm{H}$, et al. Vitrectomy with or without preoperative intravitreal bevacizumab for proliferative diabetic retinopathy: a meta-analysis of randomized controlled trials. Am J Ophthalmol. 2013;156:106-15.

19. Su L, Ren X, Wei H, Zhao L, Zhang X, Liu J, et al. Intravitreal conbercept (KH902) for surgical treatment of severe proliferative diabetic retinopathy. Retina. 2016;36:938-43.

20. Li F, Zhang L, Wang Y, et al. One-year outcome of conbercept therapy for diabetic macular edema. Curr Eye Res. 2018;43 (2):218-23.

21. di Lauro R, De Ruggiero P, di Lauro R, di Lauro MT, Romano MR. Intravitreal bevacizumab for surgical treatment of severe proliferative diabetic retinopathy. Graefes Arch Clin Exp Ophthalmol. 2010;248:785-91.

22. Holladay JT. Proper method for calculating average visual acuity. J Refract Surg. 1997;13:388-91. 
23. Ajvazi H. Proliferative diabetic retinopathy-results of the microsurgical and laser treatment. Med Arch. 2010;64:165-7.

24. Cheung N, Mitchell P, Wong TY. Diabetic retinopathy. Lancet. 2010;376:124-36.

25. Ahmadieh H, Shoeibi N, Entezari M, Monshizadeh R. Intravitreal bevacizumab for prevention of early postvitrectomy hemorrhage in diabetic patients: a randomized clinical trial. Ophthalmology. 2009;116:1943-8.

26. Kohno R, Hata Y, Moehizuki Y, Arita R, Kawahara S, Kita T, et al. Histopathology of neovascular tissue from eyes with proliferative diabetic retinopathy after intravitreal bevacizumab injection. Am J Ophthalmol. 2010;150:223-9.

27. Ip MS, Domalpally A, Hopkins JJ, Wong P, Ehrlich JS. Longterm effects of ranibizumab on diabetic retinopathy severity and progression. Arch Ophthalmol. 2012;130:1145-52.

28. Moradian S, Ahmadieh H, Malihi M, Soheilian M, Dehghan MH, Azarmina M. Intravitreal bevacizumab in active progressive proliferative diabetic retinopathy. Graefes Arch Clin Exp Ophthalmol. 2008;246:1699-705.
29. Yeh PT, Yang CM, Lin YC, Chen MS, Yang CH. Bevacizumab pretreatment in vitrectomy with silicone oil for severe diabetic retinopathy. Retina. 2009;29:768-74.

30. Arevalo JF, Maia M, Flynn HW Jr, Saravia M, Avery RL, Wu L, et al. Tractional retinal detachment following intravitreal bevacizumab (Avastin) in patients with severe proliferative diabetic retinopathy. Br J Ophthalmol. 2008;92:213-6.

31. Ishikawa K, Honda S, Tsukahara Y, Negi A. Preferable use of intravitreal bevacizumab as a pretreatment of vitrectomy for severe proliferative diabetic retinopathy. Eye (Lond). 2009;23:108-11.

32. El-Sabagh HA, Abdelghaffar W, Labib AM, Mateo C, Hashem TM, Al-Tamimi DM, et al. Preoperative intravitreal bevacizumab use as an adjuvant to diabetic vitrectomy: histopathologic findings and clinical implication. Ophthalmology. 2011;118:636-41.

33. Gupta A, Bansal R, Gupta V, Dogra MR. Six-month visual outcome after pars plana vitrectomy in proliferative diabetic retinopathy with or without a single preoperative injection of intravitreal bevacizumab. Int Ophthalmol. 2012;32:135-44. 